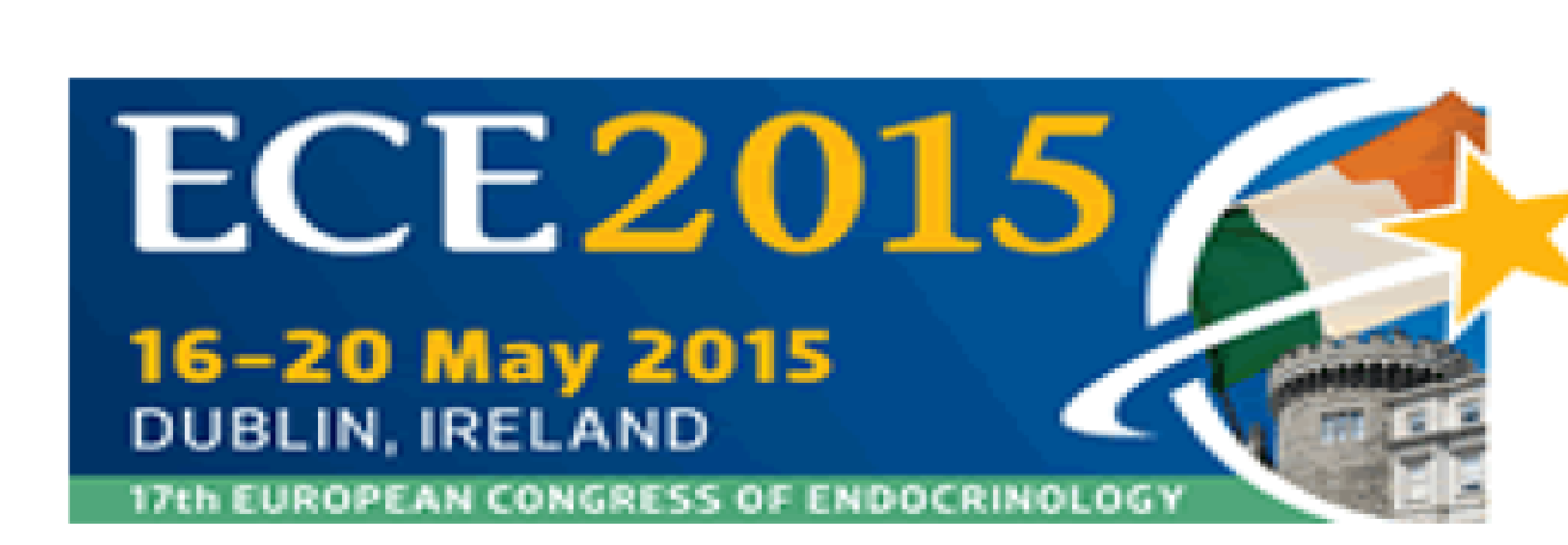

\title{
HYPERTHYROIDISM IN INTERNAL MEDICINE SERVICES IN SPAIN (2005-2012)
}

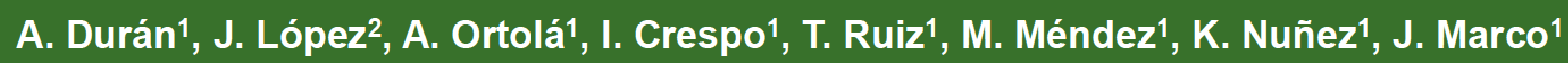

1 Hospital Clínico San Carlos, Madrid, Spain.

2 Hospital Universitario de Canarias, San Cristobal de la Laguna, Spain

\section{INTRODUCTION}

The prevalence of hyperthyroidism in patients admitted in Internal Medicine at Spanish hospitals is $0.8 \%$.

Proper diagnosis and management of hyperthyroidism in these patients is still a challenge and therefore, the analyses of the factors involved in the prognosis and survival is of interest.

\section{MATERIAL AND METHODS}

Data from the minimum-data-set of Spanish National health System were collected during 2005-2012 from discharged patients of Internal Medicine with an hyperthyrodism diagnosis. A bivariante analysis was performed in order to detect differences in the mortality rate, median age, median hospital stay, readmission rate and comorbidities among hyperthyroid and non hyperthyroid patients. A logistic regression analysis was performed using as dependent variable in-hospital mortality and sex, mean age, adjusted comorbidities measured by the Charlson's index and hyperthyroidism as independent variable. Chi square and Student-T test were performed. Data analysis used SPSS22 software.

\section{RESULTS}

TABLE 1: Prevalence of hyperthyroidism in Internal Medicine hospital inpatients

\begin{tabular}{c|c|c|} 
Hyperthyroidism & Non Hyperthyroisdism & TOTAL \\
\cline { 1 - 3 } 32,515 & $3,952,018$ & $3,984,533$
\end{tabular}$\quad \begin{array}{ll}\text { Prevalence of encoded hyperthyroidism: } \\
0.8 \% \text { in hospital inpatients }\end{array}$

TABLE 2: Baseline characteristics and comorbidities of hyperthyroid and non hyperthyroid patients

\begin{tabular}{|l|c|c|c|}
\hline & Hyperthyroidism & Non Hyperthyroidism & $\boldsymbol{p}$ \\
\hline PATIENTS & 32,515 & $3,952,018$ & \\
\hline Age (years) & 77 (SD 12.71) & 72 (SD 17.06) & $<0.001$ \\
\hline Sex (\% women) & 66 & 47.1 & $<0.001$ \\
\hline Hypertension (\%) & 42.2 & 37 & $<0.001$ \\
\hline Diabetes Mellitus (\%) & 30.6 & 27.6 & $<0.001$ \\
\hline Dyslipidemia (\%) & 16.7 & 16.5 & 0.23 \\
\hline Smoking (\%) & 6.5 & 11.1 & $<0.001$ \\
\hline Obesity (\%) & 7.6 & 7.7 & 0.545 \\
\hline Alcoholism (\%) & 1.1 & 2.4 & $<0.001$ \\
\hline Atrial fibrillation (\%) & $\mathbf{4 0 . 9}$ & $\mathbf{2 1 , 8}$ & $<0.001$ \\
\hline Congestive heart failure (\%) & $\mathbf{3 3}$ & $\mathbf{2 0 . 9}$ & $<0.001$ \\
\hline Peripheral vascular disease (\%) & 5.6 & 5.5 & 0.4 \\
\hline Coronary disease (\%) & 7.6 & 8.5 & $<0.001$ \\
\hline Cerebrovascular disease (\%) & 10.5 & 11.2 & $<0.001$ \\
\hline Chronic renal disease (\%) & 13.7 & 11.7 & $<0.001$ \\
\hline Liver disease (\%) & 2.7 & 5.5 & $<0.001$ \\
\hline Chronic pulmonary disease (\%) & 32.1 & 25.3 & $<0.001$ \\
\hline Dementia (\%) & 6 & 6.1 & 0.851 \\
\hline Neoplasia (\%) & 6.8 & 11 & $<0.001$ \\
\hline
\end{tabular}

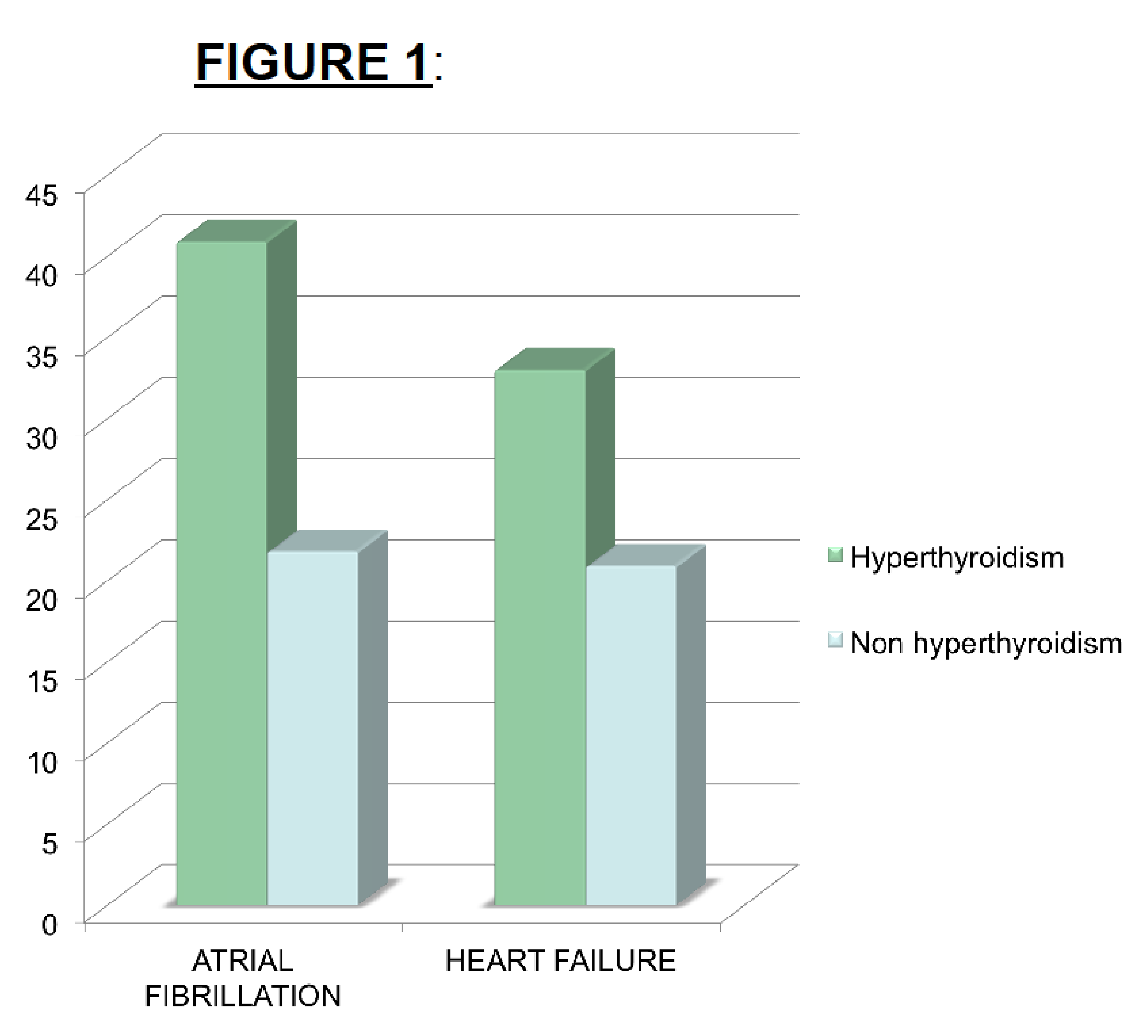

Atrial fibrillation and congestive heart failure were more common in hyperthyroid versus non hyperthyroid patients (figure 1).

\section{MORTALITY RATE:}

$\rightarrow$ The mortality rate in hyperthyroid patients was lower than in non hyperthyroid patients $(8 \%$ vs $10 \% ; p<0.001)$.

$\rightarrow$ In the logistic regression analyses adjusted by age, sex and Charlson's cormibility index, hyperthyroidism was found to be an independent factor to prevent mortality in inpatients OR 0.69 (IC: $0.66-0.74 ; p<0.0001$ ).

\section{CONCLUSIONS}

Hyperthyroid patients admitted to Internal Medicine wards have a lower mortality than non hyperthyroid patients. The frequency of women, atrial fibrillation and heart failure was higher in the hyperthyroid group. It is still unknown why hyperthyroid patients show lower mortality during admission. Perhaps other clinical and therapeutical factors, which have not been assessed through this retrospective study, have been involved with the hospital prognosis. 